\title{
Foreign Students Computer Chinese Input Problems and Teaching Strategies
}

\author{
Jiehua Zhang ${ }^{1, a}$ and Hao Bai ${ }^{1, b^{*}}$ \\ ${ }^{1}$ Beijing Language and Culture University, Beijing 100083, China; \\ bbaihao@blcu.edu.cn \\ *the corresponding author
}

Keywords: Computer; Chinese input; Teaching strategies

\begin{abstract}
Today, Chinese learning often uses computers. However, basic Chinese input does not attract enough attention from foreign students. There are also many problems with Chinese input, mainly including: 1. The basic theoretical knowledge needs to be consolidated; 2. The understanding of uniqueness of Chinese input is not deep; 3. Weak learning motivation, lack of practical exercises. These problems are universal. Through the description and analysis of these problems, advanced teaching, practice to drive learning and combination of individual and overall are put forward to improve the teaching strategies on Chinese teaching of computer and language.
\end{abstract}

\section{留学生计算机中文输入问题及教学策略}

\author{
张洁华，白浩 \\ 北京语言大学，北京 邮编：100083
}

摘要: 如今, 中文学习常常要用到计算机, 但对基础的中文输入的学习并未引起留学生足够的重视, 其中文输入也存在 许多问题, 主要为: 1. 基础理论知识有待巩固; 2. 对中文输入的独特性认识不深; 3. 学习动机较弱, 实操练习不足。这些 问题具有普遍性, 通过对这些问题的描述、分析, 提出了进阶教学、以练带学和零整结合的教学策略, 为中文计算机教学和 语言教学提供参考。

关键词: 计算机; 中文输入; 教学策略

\section{1 引言}

中文计算机教学并非近年来的新创, 早在上个世纪九十年代, 北京语言学院 (现为北京语言大学) 就 已着手规划和实施针对中国文史哲外语类专业的学生和针对来华学习各专业的外国留学生的中文计算机 课程了。然而有的留学生到了研究生阶段, 使用计算机撰写论文处理数据等仍然有很多技术上的问题。这 让笔者开始反思计算机中文输入教学的必要性和重要性。通过真实的课堂观摩, 笔者选取了在北京语言大 学汉语进修学院选修中文计算机应用的留学生作为研究对象, 分析了他们在中文输入方面的问题, 并提出 了相应的教学策略。

\section{2 教学内容设计}

卢湘鸿提出了这门课应当包含的四个特征, 其中至今仍有借鉴意义的如 “少讲理论多讲用法”, 与对 外汉语教学的经典原则 “精讲多练” 不谋而合。

北京语言大学汉语进修学院中文计算机应用课程使用的是自编教材, 供选课学生使用。教学内容大致 分为五大部分。

\section{1 计算机常用术语及办公软件操作术语}

这一部分主要介绍一些与计算机相关的专业术语, 包括硬件名称和常用的办公软件的操作术语, 方便 
后续的教学。

\section{2 中文输入法练习}

选修中文计算机应用的留学生必须具备一定的汉语基础, 本文研究对象大部分为初级下或中级上的水 平, 其中也零散分布着其他水平的学生。教师选取本学院初级教材中的课文, 要求学生使用拼音输入法在 计算机的记事本这一软件中进行中文输入练习。初步练习之后, 教师利用可记录学生打字情况的软件辅助 教学, 通过记录学生的打字速度和正确率, 并允许进行重复练习, 记录学生的进步曲线。

\section{3 中文输入和编辑排版入门}

教师以实用性为导向, 授课内容涵盖了字体、段落、插图、页眉页脚、页面设置等要点, 以经过处理 的文章格式为例, 通过直观的观摩, 用结果反向推演操作步骤, 使学生体验到学有所用, 从而增强学生的 学习动机。最后教师将输入法和文字处理结合起来, 布置实操作业, 巩固学生所学。

\section{4 数据录入和处理入门}

这一部分大致仍沿用与前几章一致的教学步骤, 从认识术语到操作步骤, 最后通过数据录入和处理练 习的课程作业来巩固学生的学习成果。

\section{5 演示文稿制作入门}

这一部分在教授课程之后, 新增了演示文稿演讲这个开放型题目, 主题为学生熟悉的生活话题, 减少 了语言障碍的干扰。教师还将这个演讲设置为小竞赛的形式, 营造竞争氛围来鼓励学生综合运用所学技能。

总体来说, 教学内容由浅入深, 从基本到进阶, 从机械练习到半机械练习再到开放性练习, 设计比较 合理，是一种可供借鉴的设计思路。

除第一章介绍术语, 中文输入贯穿了整个计算机课程的始终，只是在每个阶段所占的比重有所不同。 第二阶段的输入练习是在为后续的中文编辑排版、演示文稿制作等教学内容夯实基础, 由此可见中文输入 学习的重要性。

\section{3 留学生在中文输入过程中出现的问题}

\section{1 基础理论知识有待巩固}

不论是拼音的错误使用还是词汇的形义分离都反映了留学生汉语水平限制了他们中文计算机输入的 效率, 汉语水平是学习中文计算机应用的第一道门槛。此外还有中文计算机的基础知识欠缺, 专业术语的 听说练习不足，对术语的理解记忆欠缺等问题。

\section{2 对中文信息处理的独特性认识不深}

当今社会利用计算机辅助生活工作学习已经普及, 很多留学生在本国可以熟练进行以其母语为系统语 言的计算机操作。因此在学习中文计算机相关知识时, 常常发生迁移, 即将其母语的输入或者编辑习惯应 用在中文文本处理上，其中既有正迁移也有负迁移。学生对负迁移的重视程度不够。

\section{3 学习动机较弱, 实操练习不足}

此门课程是非语言教学的选修课, 教学重点不都是汉语知识, 通过访谈, 我们了解到, 大多选修此门 课程的学生学习动机是外部驱动。即使是想通过这们课程掌握一些中文计算机知识的学生内部动机也并不 强, 表现之一就是分配在计算机实操练习或者复习的课余时间几乎为零。教师尽量在压缩理论讲解和演示 的时长, 但是课上的时间仍是远远不够的, 加之计算机专业术语在留学生日常生活较少复现, 如果不勤加 
练习, 短时记忆难以形成长时记忆。

\section{4 学习者的个人习惯}

我们发现, 有相当一部分学生不熟悉键盘, 指法单一。输入时, 眼睛和手指配合度较差, 效率较低。 个别学生按句输入, 无法及时更正句中错字, 对输入速度和正确率都造成了较大影响。这部分学生或则以 前并未接触过计算机输入, 尚处于初学阶段; 或则已经形成了错误的输入习惯, 中文输入练习放大了这些 缺陷。

\section{4 教学策略}

我们观察到，授课教师在教授中文计算机应用这门课程时，有很多经验值得借鉴，综合笔者的思考后 可概括为以下三点。

\section{1 进阶教学}

面向留学生的中文计算机教学理论内容并不复杂, 重点是教师要关注学生的可理解性输入, 及时调整 教学内容和教学进度。学期课程开始之前, 教师已有一套完整的教学计划和安排, 这是根据以往经验制定 出来的, 如理论知识从表层到深层, 技能从单一到复合, 大致符合学生的认知规律。此外, 教师还在不同 阶段安排了难度不同的课堂任务, 既有面向所有学生的分布在不同教学阶段的任务, 也有面向不同学习能 力的学生设计的有难度梯度的练习。

汉语水平决定了中文输入的内容和评价的标准。本文的研究对象均为学习汉语半年以上一年以下的留 学生, 因此中文输入的内容也随着学生中文学习的进度而变化, 评价的标准既有一定难度达到又不会过于 超出学生的水平。如在中文输入法练习阶段, 最初教师对学生的输入速度没有要求, 只要求达到 $100 \%$ 的正 确率; 第二次输入练习使用和第一次相同的文本, 但是提出了 20 字/分钟的低要求, 大部分学生通过 1-2 次练习后可以达到; 第三次文本输入练习使用新文本, 学生已经大致熟悉了中文输入的一般操作, 此时教 师又提高了标准, 需达到 30 字/分钟。我们认为, 对于相同的文本, 练习次数越多, 机械性操作的比例越 大, 理解性输入的比例越小, 而选取相似长度和难度但不同内容的文章供学生练习则可以训练学生对于常 用字词字段的掌握使用, 进行有替换组合的理解性练习, 同时提高了速度要求, 这样就避免了学生机械记 忆, 促使学生举一反三, 掌握要领。

\section{2 精讲多练, 以练带学}

“精讲多练” 本为对外汉语教学上的核心原则, 在中文计算机的教学上二者是互通的。计算机操作本 身就是一项实用性较强的技术, 卢湘鸿甚至提倡 “不讲” 只练, 因办公软件本身为精简办公服务, 故技术 门槛不会过高, 不会对学生造成过大的负担。因此教师可适当压缩授课时长, 扩充学生实操的时长。如研 究样本中的教师在术语讲练部分, 从教师示范读、领读到学生自读、教师点读再到最后教师要求学生做术 语和实物图片的匹配练习, 就很好地体现了这一原则, 当然这还是属于语言教学的范畴, 体现在计算机技 术的学习和实操上, 也是一样的。如教师在中文输入和编辑排版入门这一部分中, 教师将本节课需要掌握 的知识按照相关程度分割成几个小块, 每讲解一个小块都会给学生自主练习的时间。全部小块都讲练完毕 后, 教师会演示一篇完整的中文输入和排版样本的编辑方式。演示完毕再要求学生进行文章输入和编辑练 习, 教师再根据学生出现的问题进行个别辅导, 如果问题较为集中普遍则进行统一讲解。讲解的时间不超 过一次授课时长的四分之一。 


\section{3 零整结合}

“整” 指的是面向全体学生的教学, “零”指的是针对学生出现的特殊情况进行的教学调整。“零整结 合”体现的是将把控全局和关注个体相结合的教学理念。

尽管教师制定了较为规范合理的教学计划, 但也可能会出现非预期的情况。如在中文输入的练习过程 中, 有位学生基础非常好, 在渡过了对中文输入的适应初期后, 输入速度进步较快, 最快可达到 77 字/分 钟。教师便根据后续的教学内容, 布置了一些符合其计算机水平的小任务, 但并不会占用其全部的剩余课 堂时间, 使学生既能得到一定时间的休息又不至于因没有课堂指令而无聊。相反, 也有学生初次接触计算 机, 对键盘上的字母分布比较陌生, 对文章的熟悉度也比较低, 输入速度只能达到 5-7 字/分钟, 教师适 当地给学生一些建议, 包括指法分配的练习方法, 基础的字母、音节的练习方法等, 通过新任务的布置, 调整对该学生的评价标准以减少学生的焦虑感, 更扎实地掌握计算机中文输入的基本功, 为尽快进入正式 中文输入做好准备。

在中文输入和编辑排版入门这一部分, 学生如果提前较多时间完成课堂任务, 教师会根据学生完成情 况适当提高要求或安排预习任务等。

\section{5 结论与启示}

本文从问题入手，归纳了以北京语言大学汉语进修学院中文计算机应用课程中的巴基斯坦学生为例的 三个具有代表性的问题, 提出了相应的教学策略, 以期对类似的教学有一定的启发意义。中文计算机应用 作为一门应用型的课程, 应当引起更多的重视。本文现状描述有余, 理论根基不深, 有待进一步研究。

\section{6 致谢}

本文受 2017 年北京语言大学校级新开课项目资助, 项目编号为: C180102。

\section{Acknowledgements:}

This article is funded by the school-level new project of Beijing Language and Culture University in 2017. The project number is: $\mathrm{C} 180102$.

\section{参考文献:}

[1] 卢湘鸿. 论我国高等学校文史哲外语类计算机应用课的设置 $[J]$. 语言教学与研究，第一期：128-129.

[2] 张晓卉. 面向留学生的计算机中文输入方法的选择与教学方法探析 $[\mathrm{J}]$. 文教资料, 第 17 期：146.

[3] 张文艳. 《中文Word》教学浅议 $[J]$. 吕梁教育学院学报，第 92 期： 76 .

[4] 张媛．谈计算机应用技术与中文教学的整合 $[J]$ ．民营科技，2008 年第五期：79

[5] 周玉珊. 浅议中文信息处理技术在外汉语教学中的运用 [J]. 北方文学, 2011 年 11 月刊： 136

[6] 王建勤．第二语言的习得研究 [M]．2009 年 8 月第 1 版．北京：商务印书馆，2015

[7] 白浩. 来华留学生预科生计算机基础课教学研究一一以北京语言大学为例 [M]//王瑞烽 王俊毅. 汉语进修教育多 角度研究. 2015 年 6 月第 1 版. 北京：中国书籍出版社，2015

\section{References}

[1] X.H.Lu: The setting of computer application course for Literature, History, Philosophy and Foreign Languages Class in Chinese Colleges and Universities[J], Language Teaching and Linguistic Studies, No.1, p:128-129.(In Chinese)

[2] X.H.Zhang: The Selection and Teaching Methods of Computer Chinese Input Method for Foreign Students[J], Data of Culture and Education, No.17m p:146.(In Chinese)

[3] W.Y.Zhang: A Brief Discussion on the Teaching of "Chinese Word" [J], Journal of Lvliang Education Institute, No.92, p: 76.(In Chinese) 
[4] Zhang: Integration of Computer Application Technology into Chinese Teaching[J], Private Science and Technology(2008)No.5, p: 79. (In Chinese)

[5] Y.S.Zhou: A Brief Discussion on the Application of Chinese Information Processing Technology in Teaching Chinese as a Foreign Language[J], Northern Literature, 2011,11,p:136.(In Chinese)

[6] J.Q.Wang: Study on the Acquisition of Second Language[M], 2009, 8, No.1, Beijing: Commercial Press, 2015(In Chinese)

[7] H.Bai: A Study on the Basic Computer Course Teaching of Preppies of International Students in China -Taking Beijing Language and Culture University as an Example[A]// R.F.Wang and J.Y.Wang: A Multi-Angle Study of Chinese Advanced Education,2015,6 No.1, Beijing: Chinese Book Publishing House(2015).(In Chinese) 